\title{
Tailored dispersive elements for adapted spectrometric sensing
}

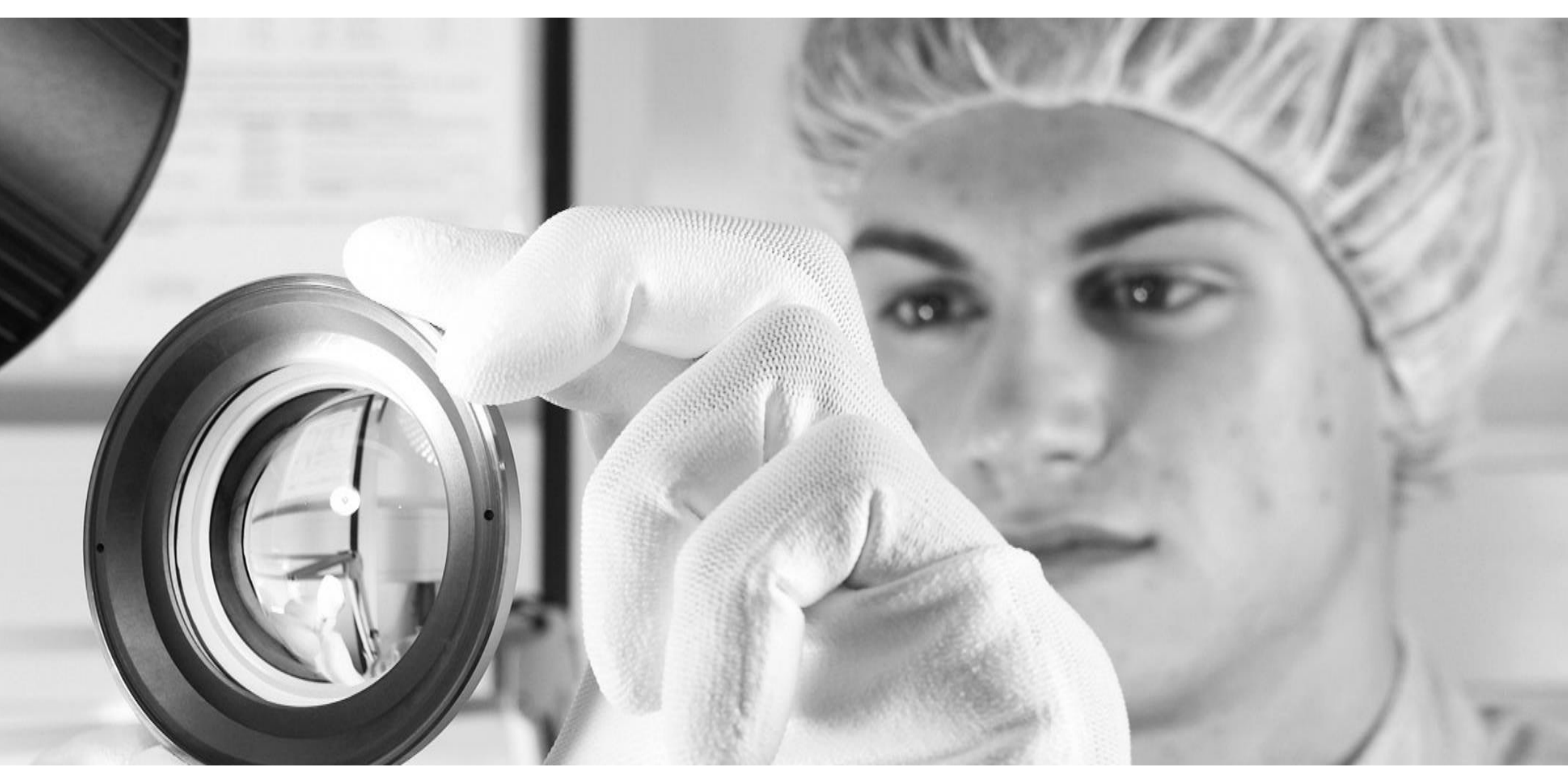

Peter Triebel, Torsten Diehl, Tobias Moeller, Alexandre Gatto, Dennis Lehr, Alexander Pesch, Lars H. Erdmann, Matthias Burkhardt, Alexander Kalies 


\section{Agenda}

1 Motivation

2 Grating Manufacturing

3 Optimization of the Grating Manufacturing Process

4 Results - Line density and wavefront measurements

5 Examples

6 Summary 


\section{Agenda}

1 Motivation

2 Grating Manufacturing

3 Optimization of the Grating Manufacturing Process

4 Results - Line density and wavefront measurements

5 Examples

6 Summary 


\section{Applications and types of gratings}

Difftraction gratings for various types of applications and spectrometer, e.g. space gratings, spectrometers and gratings for industrial applications
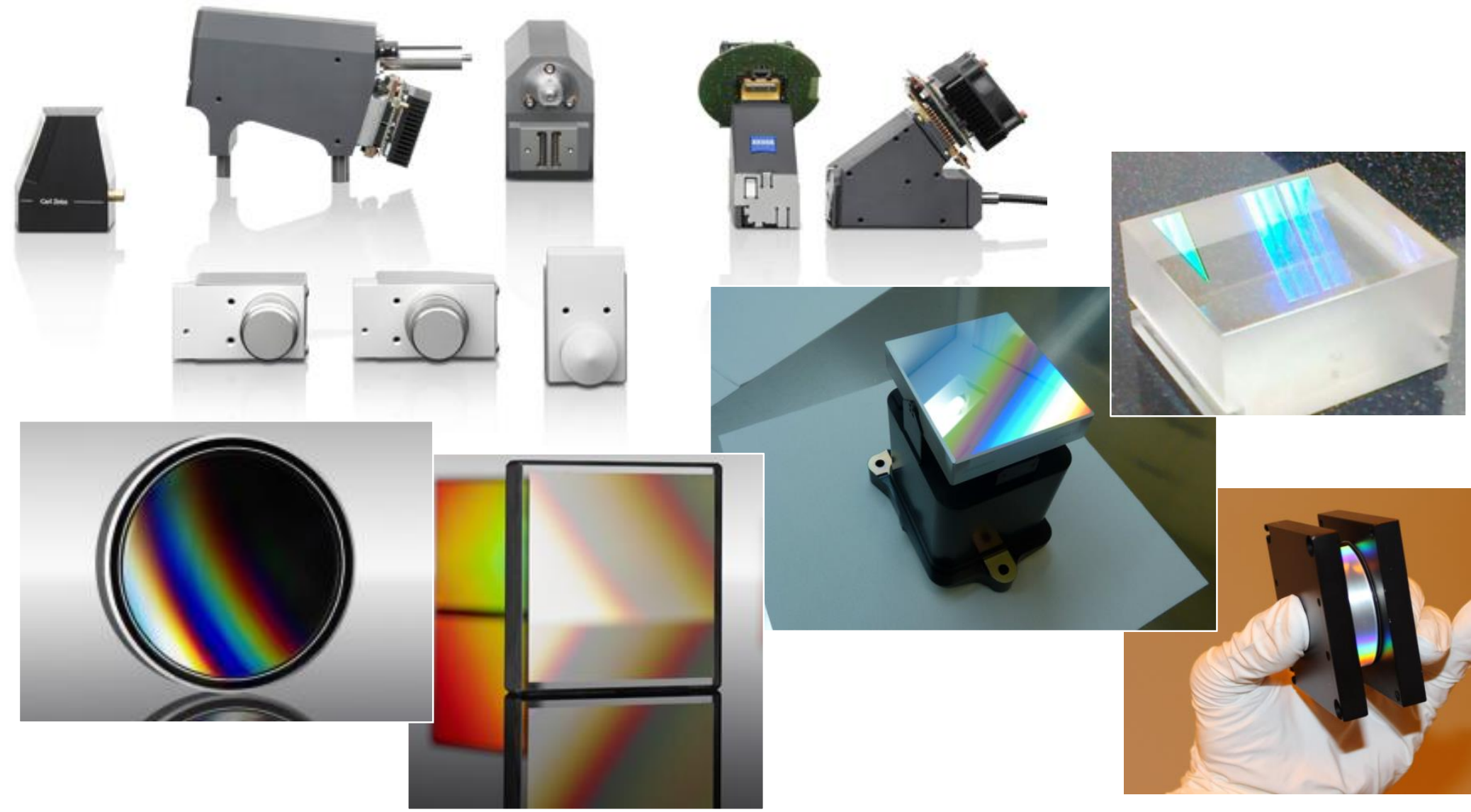


\section{Key requirements for diffraction gratings for space applications}

Major requirements:

- High efficiency in the defined/customized wavelength range

- Aberration and imaging performance including the wavefront error of the diffracted order, e.g. customized wavefront aberrations in order to enhance to optical performance of spectrometer

- Tailored efficiency characteristics e.g. for avoiding detector saturation

- Low polarization sensitivity

- Low straylight of the microstructure and the substrate in and around (FOV) the application order

- Groove alignment and adapted substrate dimensions / features for the support of AIT

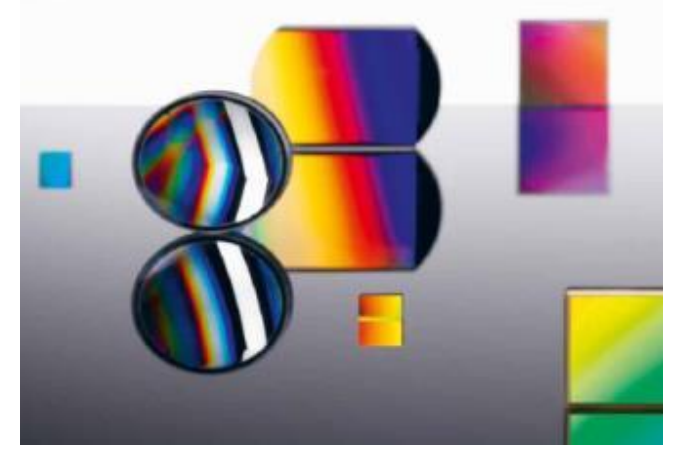




\section{Agenda}

1 Motivation

2 Grating Manufacturing

3 Optimization of the Grating Manufacturing Process

4 Results - Line density and wavefront measurements

5 Examples

6 Summary 


\section{Recent Grating Manufacturing Technologies}

\section{Serial Grating Generation}

Technologies

e-Beam
Direct-Laser-Writing
Ruling

Pattern

Generation

Grating line or sub-aperture generation based on sub-grids / sub-apertures

Types of substrates

\section{Plane substrates,}

Substrates are dedicated to the microstructuring machine

Characteristics
Sub-wavelength / effective media capability's.

\section{Parallel Grating Generation}

Holography

Complete clear aperture will be exposed

plane, curved (convex, concave or free form shape) substrates, aspheres prisms Holographic setup will be adapted Adaption of the wavefront corrections 


\section{Holographic Exposure Setup of Interference}

\section{Lithography for Symmetric and Blaze profiles}
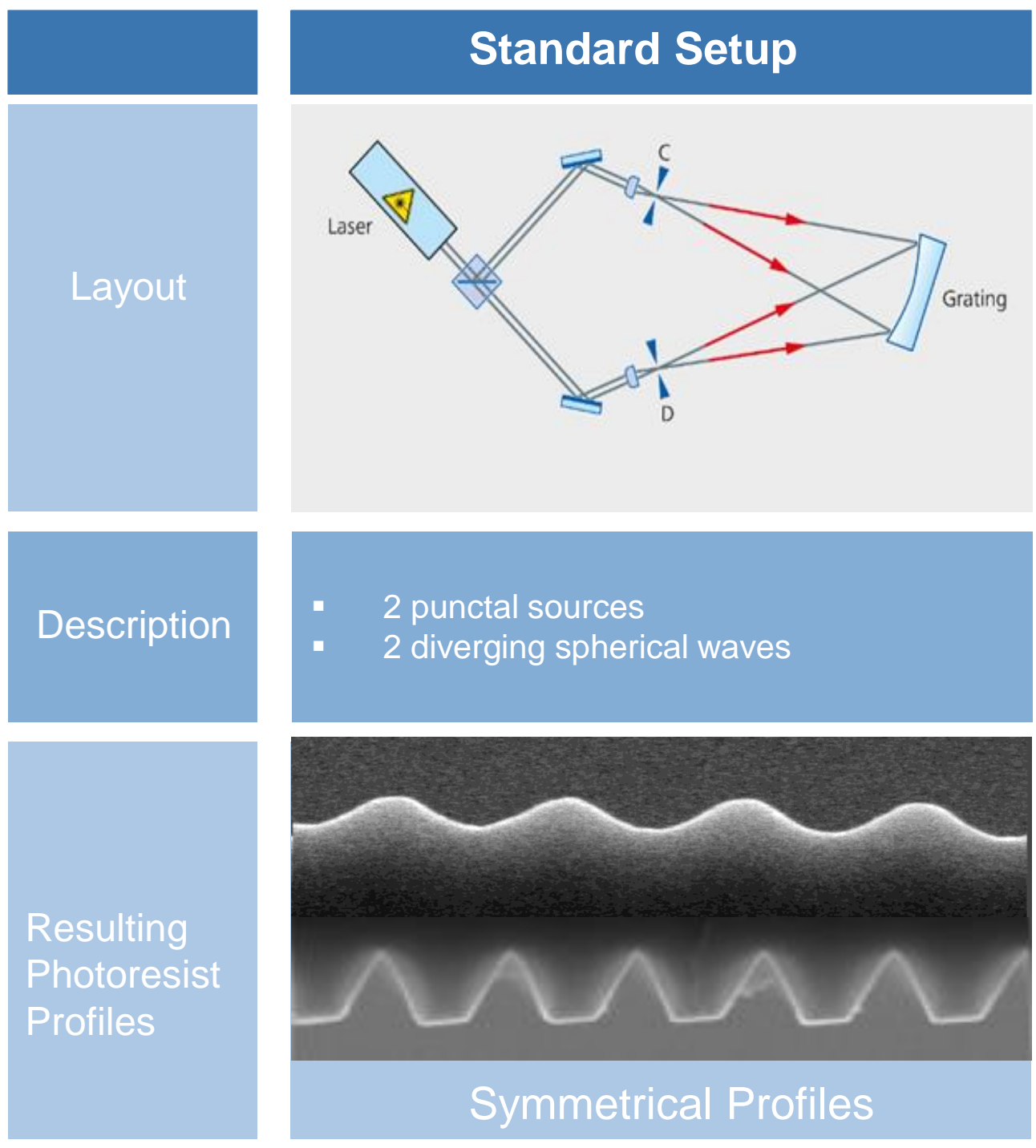

Symmetrical Profiles

\section{ZEISS Approach}

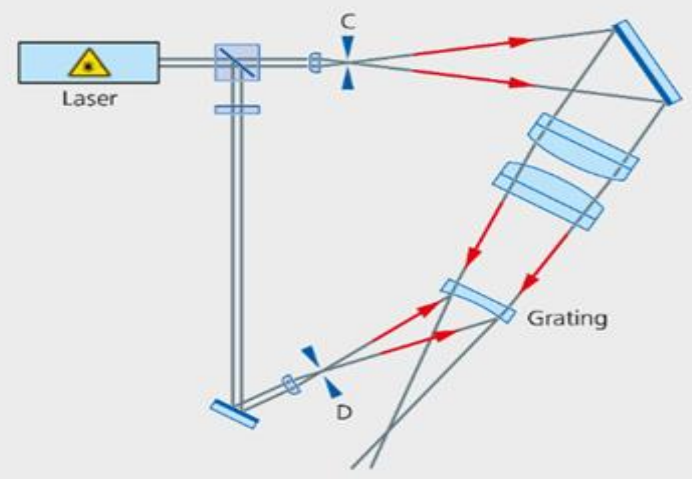

- 2 punctual sources

- Converging and diverging spherical waves leading to a blaze profile in the photoresist

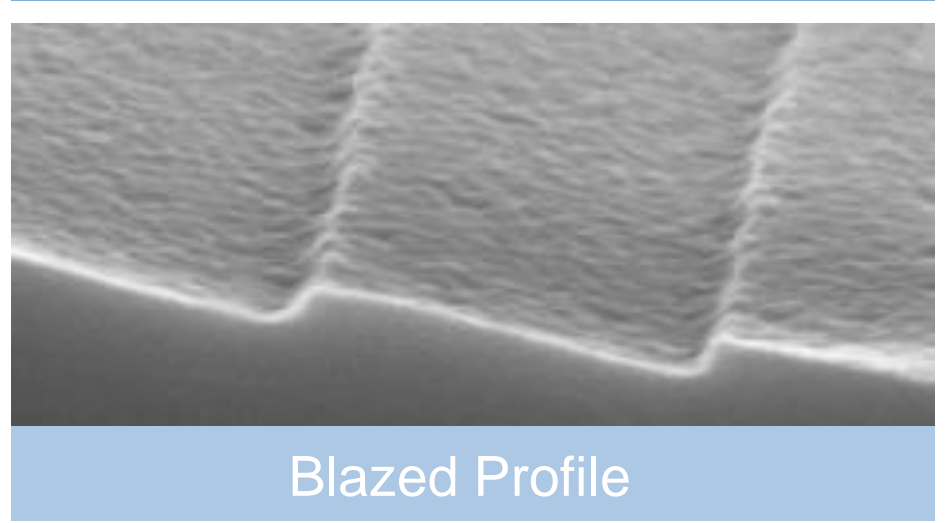




\section{Technological process for manufacturing of a holographic blazed type grating}

air

Defines:

Principal groove shape

(blaze or symmetric)

\section{Defines:}

Actual groove shape

Profile smoothness
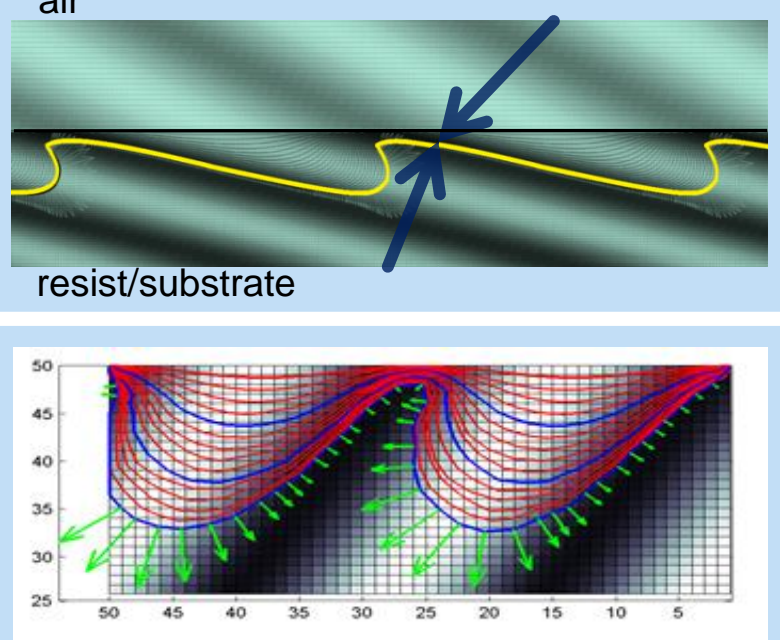

Development

(Wet-Chemical)
Defines:

Groove depth

Blaze angle
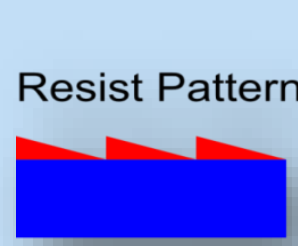

Beam Etching

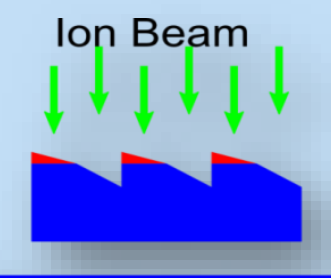

Monolithic

Grating

\section{Grating}

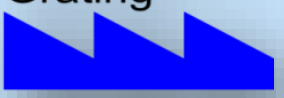

Ion Beam

Defines:

Groove depth

Blaze angle

Tip-cut

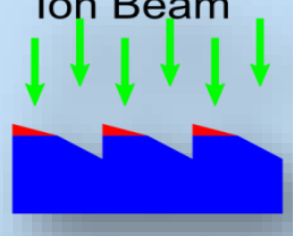

Monolithic

Grating

loop

Resist Pattern

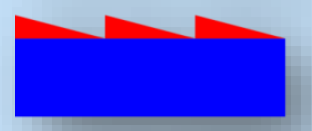

$\checkmark$

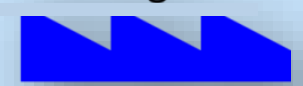




\section{Agenda}

1 Motivation

2 Grating Manufacturing

3 Optimization of the Grating Manufacturing Process

4 Results - Line density and wavefront measurements

5 Examples

6 Summary 


\section{Simulation of the etching process from photo- resist into fused silica}

Example: Blaze-Grating 1200 L/mm
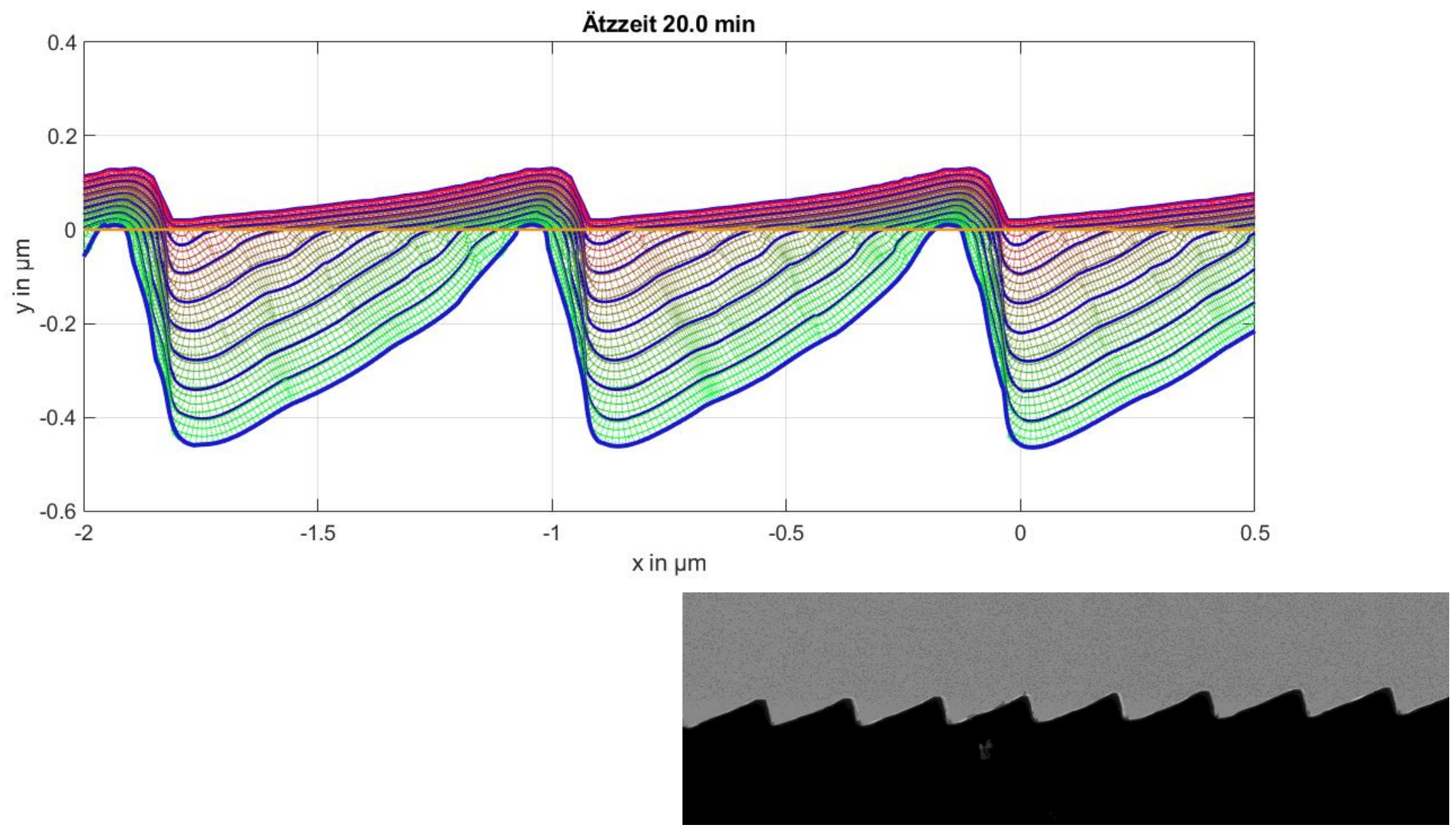


\section{Agenda}

1 Motivation

2 Grating Manufacturing

3 Optimization of the Grating Manufacturing Process

4 Results - Line density and wavefront measurements

5 Examples

6 Summary 


\section{Wavefront optimization and measurement}
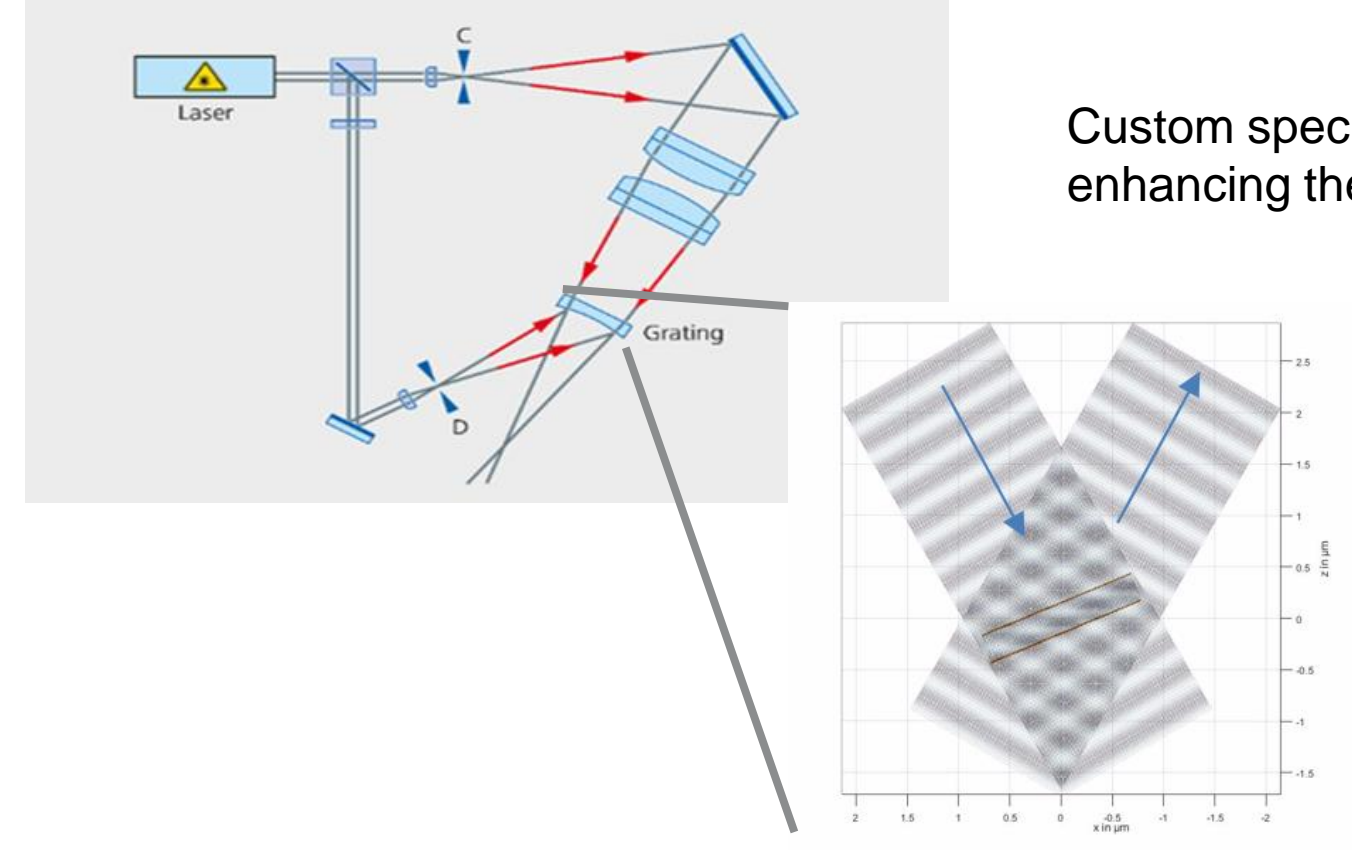

Custom specific wavefront aberrations for enhancing the spectrometer performance

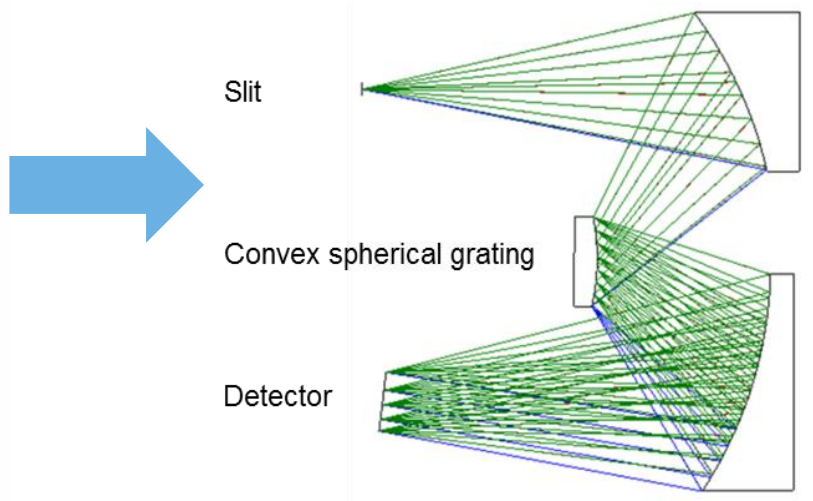

Mirror 1

- Additional optical design parameters with various degrees of freedom can be used for the implementation of aberration corrected gratings and VLS (variable line space) gratings

- Commonly a recording setup can be derived from any customers grating description based on substrate surface figure and line density distribution.

- Additionally, the potential for bending the lines and/or use higher order coefficients in case of a VLS grating can be employed for achieving enhanced focusing properties or imaging quality. 


\section{Moire pattern and calculated wavefront distortion generated by test setup for an Offner grating}
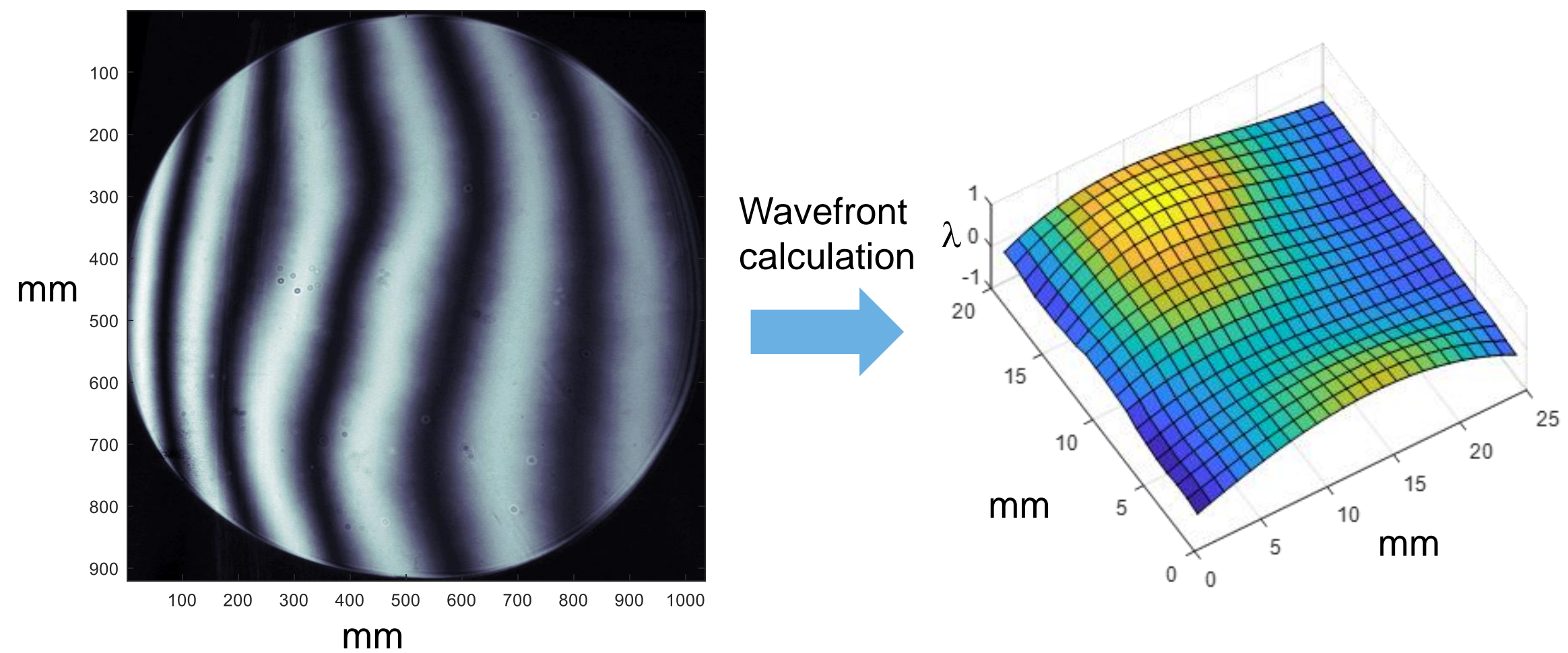

- Projection of the convex grating and a virtual plane grating generates the Moire Pattern @ 450nm.

- Here one can get a benefit from the fact that an equidistant and parallel line distribution is given if the gratings lines are projected onto a tangential plane through the vertex of the grating substrate

- Accuracy better than lambda/20 


\section{Moire pattern and calculated wavefront distortion generated by test setup for an VLS grating}
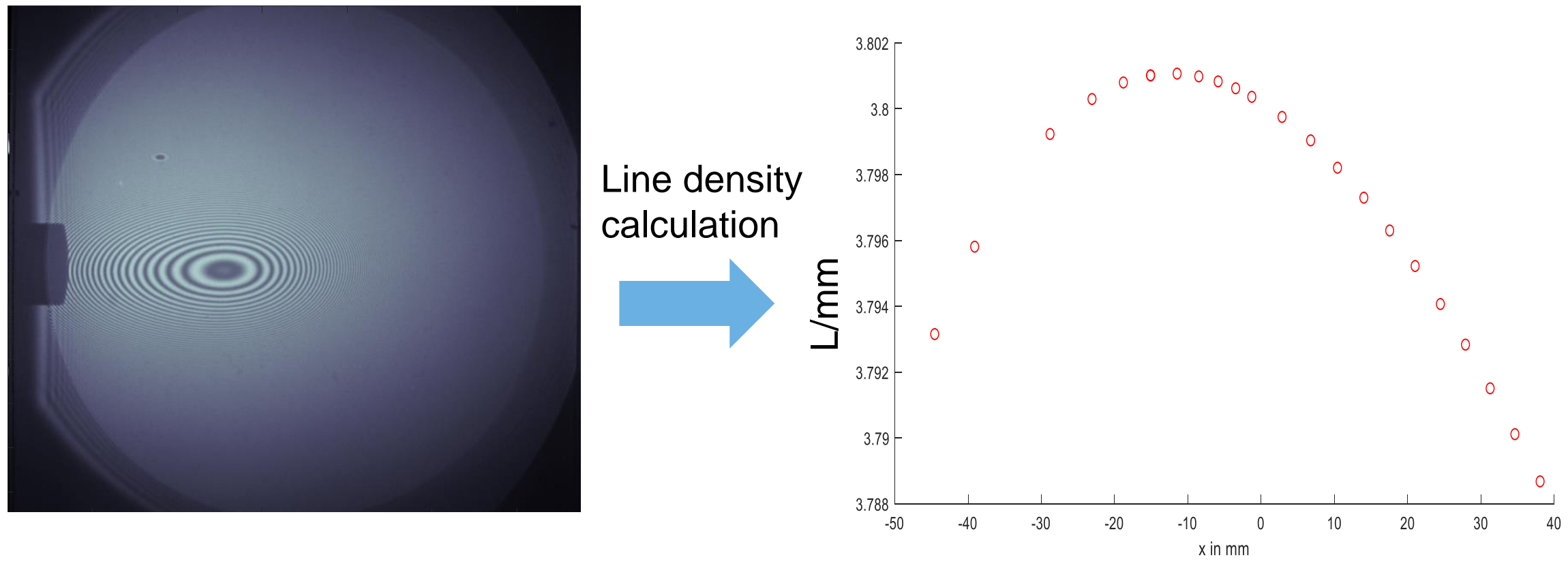

- The presented measurement results of existing gratings show the capability of measuring line density distributions or wavefront aberrations are a standard measurement technique developed at ZEISS during the past years. 


\section{Agenda}

1 Motivation

2 Grating Manufacturing

3 Optimization of the Grating Manufacturing Process

4 Results - Line density and wavefront measurements

5 Examples

6 Summary 


\section{Example 1 - Spectrometer Gratings and experimental setup - Offner Spectrometer}

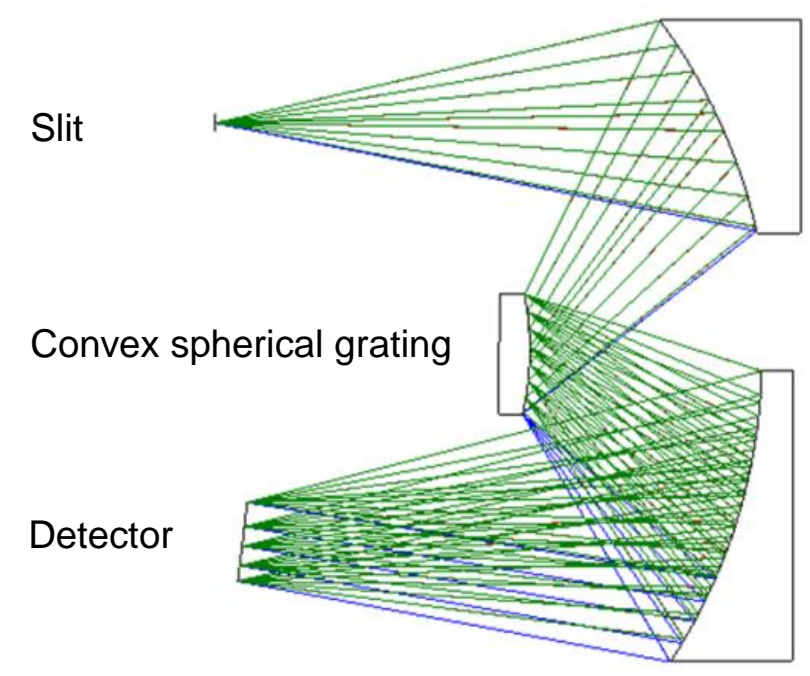

Mirror 1

Mirror 2
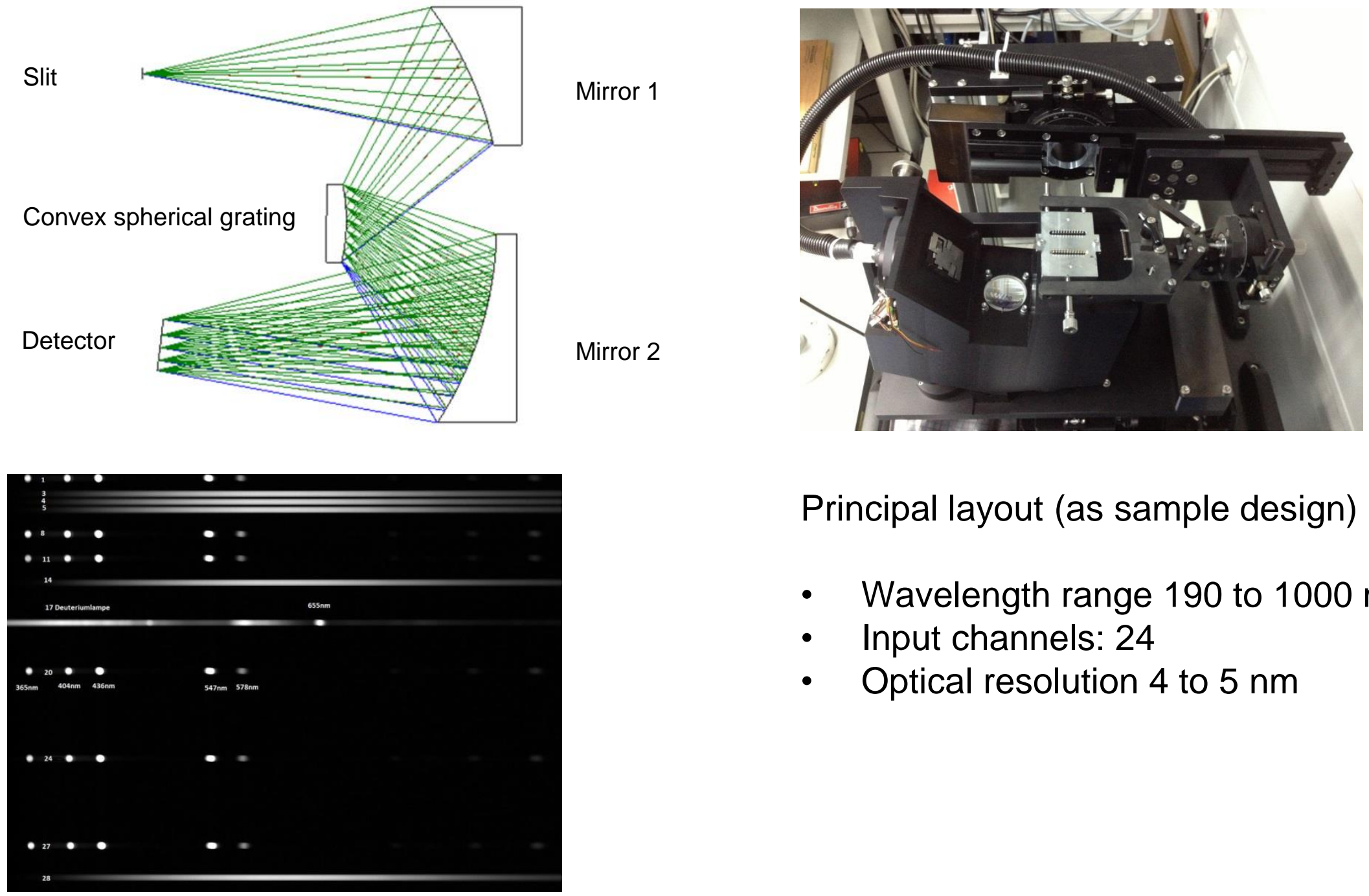

Principal layout (as sample design)

- Wavelength range 190 to $1000 \mathrm{~nm}$

- Input channels: 24

- Optical resolution 4 to $5 \mathrm{~nm}$ 


\section{Example 3: Modulated blaze grating for wide bandwidth applications}
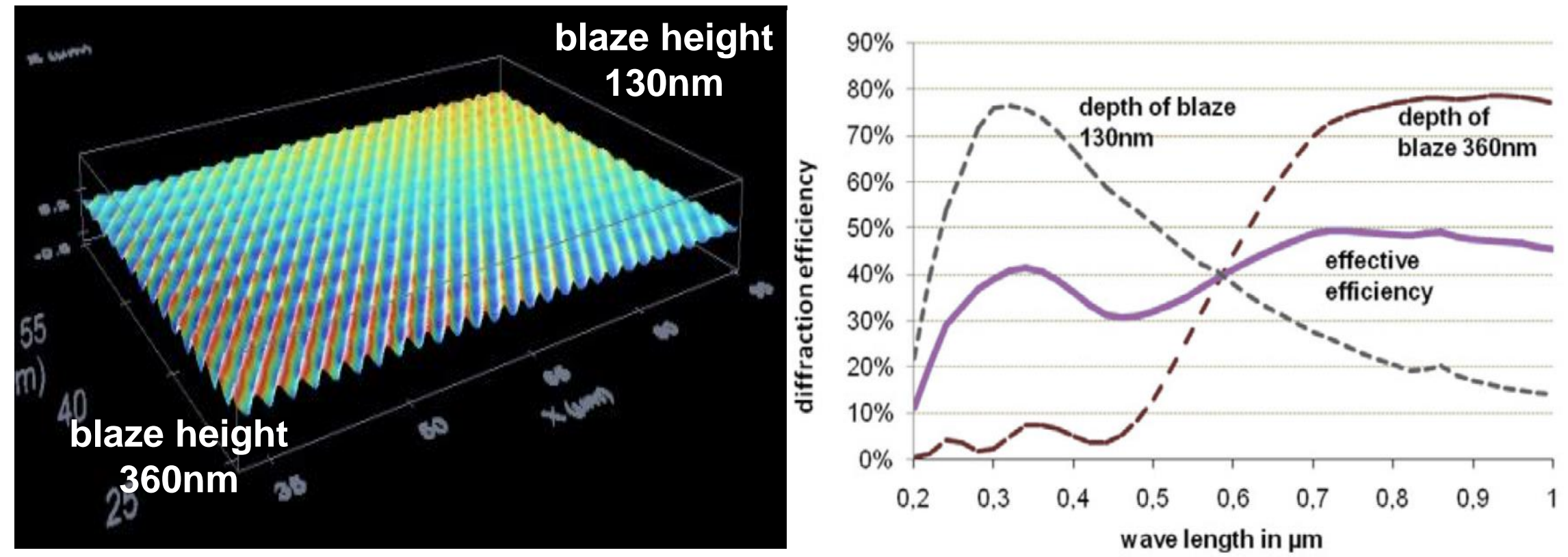

- Variation of the blaze over the grating surface area without having transition areas

- Improving grating efficiency for a wide wavelength range 


\section{Example 3: Modulated blaze grating for wide bandwidth applications}

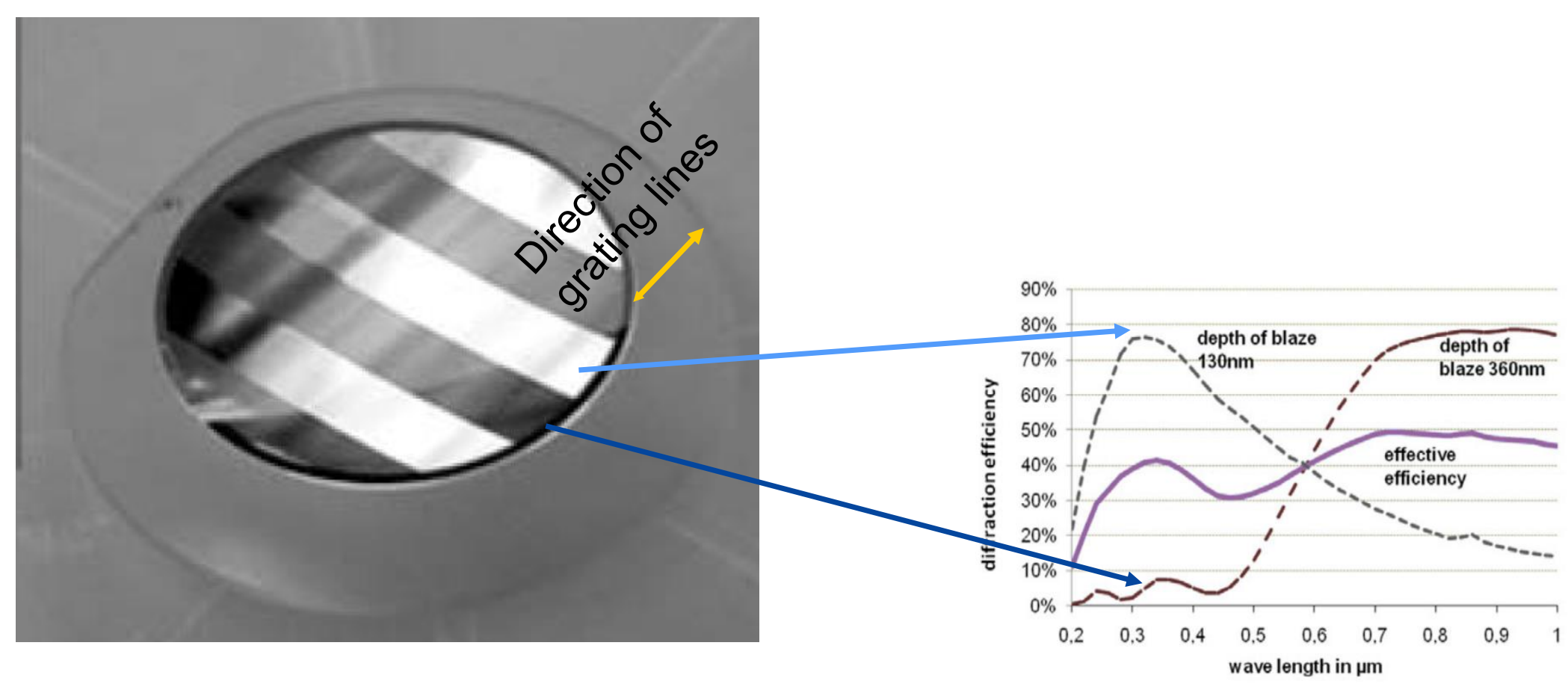

- No loss of spectral resolution (imaging quality) due to still coherent grating structures with tiling orthogonal to grating lines

- Tiling adaptable to varying intensity distributions in pupil plane 


\section{Agenda}

1 Motivation

2 Grating Manufacturing

3 Optimization of the Grating Manufacturing Process

4 Results - Line density and wavefront measurements

5 Examples

6 Summary 


\section{Conclusion}

- The results show approaches of holographic setups and their adaption for the generation of tailored wavefront aberrations over the clear aperture.

- The presented results are reporting the measurement technique and wavefront aberration on a classical Offner type grating. The accuracy of those measurement technique is around lambda/20 or even better using the existing test setup.

- Beside the measurement of the classical Offner-type grating a VLS grating is one of main application fields of the presented measurement technique, which allows an accurate verification of the line density distribution and its comparison to nominal line density distribution defined by the optical design of the spectrometer system.

- Also shown in this paper is the capability of the manufacturing of blazed Offnertype and blazed VLS gratings for sophisticated spectrometer systems in remote sensing applications. 
ZEINYS 